Short communication

\title{
Exploiting knowledge of jump-up and jump-down frequencies to determine the parameters of a Duffing oscillator
}

\author{
Roszaidi Ramlan a,*, Michael J. Brennan ${ }^{\mathrm{b}}$, Ivana Kovacic ${ }^{\mathrm{c}}$, Brian R. Mace ${ }^{\mathrm{d}}$, \\ Stephen G. Burrow ${ }^{\mathrm{e}}$

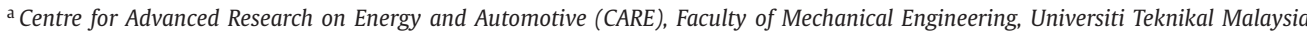 \\ Melaka, Hang Tuah Jaya, 76100 Durian Tunggal, Melaka, Malaysia \\ ${ }^{\mathrm{b}}$ Department of Mechanical Engineering, UNESP, Ilha Solteira, SP 15385-000, Brazil \\ ${ }^{c}$ Centre of Excellence for Vibro-Acoustic Systems and Signal Processing, Faculty of Technical Sciences, University of Novi Sad, \\ 21000 Novi Sad, Serbia \\ d Department of Mechanical Engineering, University of Auckland, Auckland 1142, New Zealand \\ e Department of Aerospace Engineering, University of Bristol, BS1 1TR Bristol, United Kingdom
}

\section{A R T I C L E I N F O}

\section{Article history:}

Received 2 October 2015

Revised 31 December 2015

Accepted 15 January 2016

Available online 27 January 2016

\section{Keywords:}

Duffing oscillator

Base excitation

Jumps

Cubic stiffness coefficient

Viscous damping coefficient

\begin{abstract}
A B S T R A C T
This work concerns the application of certain non-linear phenomena - jump frequencies in a base-excited Duffing oscillator - to the estimation of the parameters of the system. First, approximate analytical expressions are derived for the relationships between the jump-up and jump-down frequencies, the damping ratio and the cubic stiffness coefficient. Then, experimental results, together with the results of numerical simulations, are presented to show how knowledge of these frequencies can be exploited.
\end{abstract}

(c) 2016 Elsevier B.V. All rights reserved.

\section{Introduction}

The appearance of nonlinear phenomena is often perceived as dangerous, with a general tendency to avoid or control them. This has led to research with different approaches and tools being developed to limit the possible detrimental effects from such phenomena (see, for example, [1-3], and the references cited therein). However, the nonlinear dynamics of today is experiencing a profound change, as recent investigations have shown that nonlinear phenomena can be used to good effect. This strategy has beneficially affected different fields in science and engineering, such as vibration isolation [1,4-6], energy harvesting [7-9], micro- and nano-electro-mechanical systems [10,11].

The novel application of nonlinear phenomena presented in this paper also contributes to this trend and is related to one of the archetypical nonlinear oscillators - the Duffing oscillator [12], whose restoring force $f(u)$ consists of a linear and cubic term, and is given by

$$
f(u)=k_{1} u \pm k_{3} u^{3},
$$

where $u$ is displacement and $k_{1}$ and $k_{3}$ are the linear and cubic stiffness coefficients, respectively. It is known that this restoring force in an unforced, undamped oscillator yields a nonlinear relationship between the amplitude of displacement

\footnotetext{
* Corresponding author. Tel.: +60 6234 6891; fax: +60 62346884 .

E-mail address: roszaidi@utem.edu.my (R. Ramlan).
} 


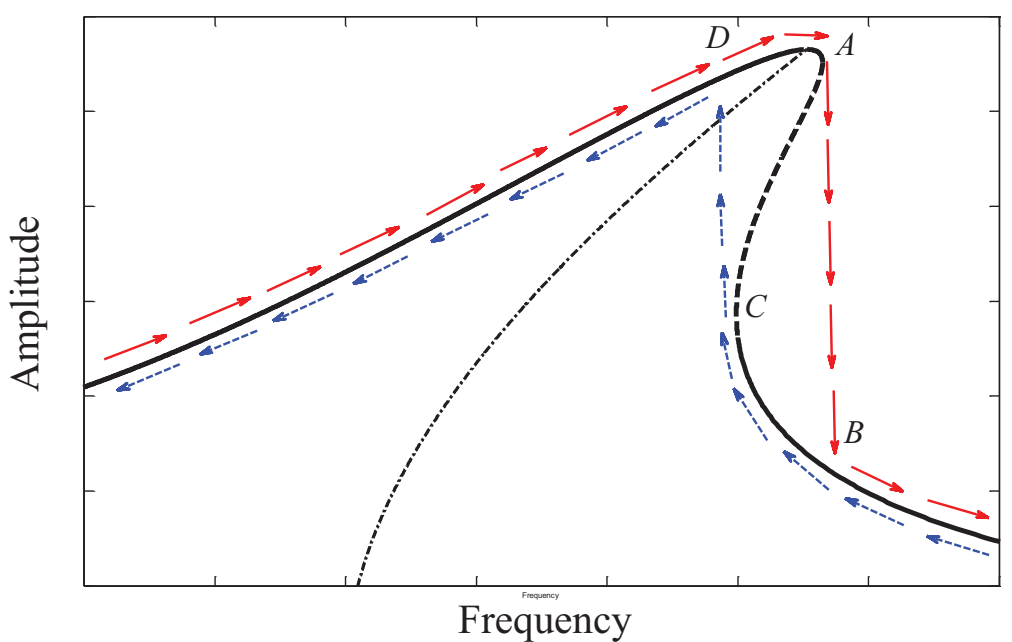

Fig. 1. Typical FRC for a hardening Duffing oscillator (the black dashed-dotted line represents the backbone curve, the black solid line represents the stable branch, while the black dashed line represents the unstable branch; red and blue arrows indicate hysteretic behaviour). (For interpretation of the references to colour in this figure legend, the reader is referred to the web version of this article.)

and the natural frequency. When presented graphically, this relationship gives the so-called backbone curve (see Fig. 1 and the dashed-dotted line plotted therein). The backbone curve bends to the right for the hardening Duffing oscillator, which has a positive cubic nonlinearity in Eq. (1.1) and to the left for the softening Duffing oscillator, which has a negative cubic nonlinearity in Eq. (1.1). When the Duffing oscillator is forced, the hysteresis phenomenon occurs [12], which is indicated by the arrows shown in Fig. 1 on the frequency response curve (FRC) of a hardening Duffing oscillator. The solid and dashed arrows depict that the amplitude of the response changes in a different way when the frequency is increased or decreased. Sudden changes of the amplitude occur from Point $A$ to Point $B$ (this represents a jump-down), while the jump-up occurs from Point $C$ to Point $D$. The corresponding jump frequencies are the boundaries of the region with multiple stable solutions on the branches of the FRC (the black solid line in Fig. 1 represents the stable branch, and the black dashed line the unstable branch). Although the jump phenomena are usually seen as undesirable, this study shows how knowledge of the frequencies when these jumps appear can be exploited. The results presented are the continuation of recent investigations concerned with the derivation of the expressions for the jump-up and jump-down frequencies for externally excited Duffing oscillators [13] and their use for the estimation of certain system parameters in such systems [14], while in this work, these expressions, experimental verification and numerical comparisons are given for a base-excited Duffing oscillator.

\section{Analysis}

Consider a damped base-excited Duffing oscillator governed by the equation of motion given by

$$
m \ddot{u}+c \dot{u}+f(u)=-m \ddot{z},
$$

where $m$ is a mass connected to a parallel combination of a viscous damper with the damping coefficient $c$ and a nonlinear spring with the characteristic given by Eq. (1.1). Note that the hardening case is considered here the case with a positive sign in Eq. (1.1). The system is excited by a harmonic base displacement $z=Z \cos \omega t$. If $u=x-z$ is the relative displacement between that of the mass $x$ and the base $z$, Eq. (2.1) can be written in non-dimensional form as

$$
\hat{u}^{\prime \prime}+2 \zeta \hat{u}^{\prime}+\hat{u}+\gamma \hat{u}^{3}=\Omega^{2} \cos (\Omega \tau),
$$

where $\hat{u}=u / Z$ is the non-dimensional relative displacement, and where the following non-dimensional parameters have been introduced: $\gamma=k_{3} Z^{2} / k_{1}, \zeta=c / 2 m \omega_{n}, \omega_{n}=\sqrt{k_{1} / m}, \Omega=\omega / \omega_{n}, \tau=\omega_{n} t,(\ldots)^{\prime}=d(\ldots) / d \tau$. Of particular interest for the subsequent analysis are the cubic stiffness coefficient $\gamma$ and the damping ratio $\zeta$.

\subsection{Approximate expressions for the jump-up and jump-down frequencies}

The expression for the FRC of the system is computed using the Harmonic Balance method by assuming that the solution to the equation has the form of $\hat{u}=\hat{U} \cos (\Omega \tau+\phi)$ in which the higher harmonics are neglected [13]. When the damping is 
small, such that $\zeta^{2}<<1$, the frequency-amplitude relationship is given by

$$
\Omega_{1,2} \approx\left(\frac{3 \gamma \hat{U}^{4}+4 \hat{U}^{2} \pm \hat{U}\left(\left(3 \gamma \hat{U}^{2}+4\right)^{2}-64 \hat{U}^{2} \zeta^{2}-48 \gamma \zeta^{2} \hat{U}^{4}\right)^{\frac{1}{2}}}{4\left(\hat{U}^{2}-1\right)}\right)^{\frac{1}{2}}
$$

where the subscripts 1 and 2 refer to the resonant and non-resonant branches of the FRC, respectively. The expression for the jump-up frequency $\Omega_{u}$ is determined from Eq. (2.3) and the condition of the existence of a vertical tangent $d \Omega_{1}(\zeta=0) / d \hat{U}=0[13]$, which gives

$$
\Omega_{u} \approx 1+\frac{27}{32} \gamma^{\frac{1}{3}}
$$

The jump-down frequency $\Omega_{d}$ is calculated by considering when the two branches of the FRC meet, i.e. when the expression in the middle brackets in Eq. (2.3) is zero. This condition yields the value of $\hat{U}$, which is substituted back in Eq. (2.3) to give

$$
\Omega_{d} \approx \frac{1}{\left(1-\frac{3 \gamma}{(4 \zeta)^{2}}\right)^{\frac{1}{2}}}
$$

Eqs. (2.4) and (2.5) imply that the jump-up frequency depends only on the cubic stiffness coefficient and not on the damping ratio, while the jump-down frequency depends on both the cubic stiffness coefficient and the damping ratio. Rearranging and combining Eqs. (2.4) and (2.5) gives the expressions for the stiffness coefficient and the damping ratio, respectively, as

$$
\begin{aligned}
& \gamma \approx 2^{6}\left(\frac{2}{3}\right)^{9}\left(\Omega_{u}-1\right)^{3} \\
& \zeta \approx 2^{\frac{3}{2}}\left(\frac{2}{3}\right)^{4} \frac{\Omega_{d}\left(\Omega_{u}-1\right)^{\frac{3}{2}}}{\left(\Omega_{d}^{2}-1\right)^{\frac{1}{2}}}
\end{aligned}
$$

It can be seen that, the estimate of the nonlinearity depends on the jump-up frequency only while the damping ratio is a function of both the jump-up and the jump-down frequencies.

\subsection{Sensitivity of stiffness coefficient and damping ratio on the jump frequencies}

The jump-down frequency can be obtained experimentally by stepping the excitation frequency from a low frequency to a high frequency in small frequency increments, and vice versa for the jump-up frequency. Inevitably, errors appear in the estimates of these frequencies, which tend to be underestimated because of the system dynamics. The estimated jumpup and jump-down frequencies are given by $\hat{\Omega}_{u}$ and $\hat{\Omega}_{d}$, respectively, and the difference between the true and estimated values of the jump-up and jump-down frequencies are given by $\xi$ and $v$, respectively. Thus $\hat{\Omega}_{u}=\Omega_{u}-\xi$, which results in an estimate of the cubic stiffness coefficient given by

$$
\hat{\gamma} \approx 2^{6}\left(\frac{2}{3}\right)^{9}\left[\left(\Omega_{u}-1\right)-\xi\right]^{3}
$$

Expanding and re-arranging Eq. (2.8) yields

$$
\hat{\gamma}=\gamma\left[1-\frac{3 \xi}{\Omega_{u}-1}+\frac{3 \xi^{2}}{\left(\Omega_{u}-1\right)^{2}}-\frac{\xi^{3}}{\left(\Omega_{u}-1\right)^{3}}\right],
$$

where $\gamma$ is the true value of the cubic stiffness coefficient given in Eq. (2.6). It can be seen in Eq. (2.9) that higher powers of $\xi$ are included. These higher order terms cannot necessarily be ignored because the denominator $\Omega_{u}-1$ may be small compared to 1 for the values used in this study. Thus the estimate of the cubic stiffness coefficient is potentially sensitive to the errors in the estimated jump-up frequency.

Now, $\hat{\Omega}_{d}=\Omega_{d}-v$, so from Eq. (2.7) the estimated damping ratio, $\hat{\zeta}$ is given by

$$
\hat{\zeta} \approx 2^{\frac{3}{2}}\left(\frac{2}{3}\right)^{4} \frac{\left(\Omega_{d}-v\right)\left[\left(\Omega_{u}-1\right)-\xi\right]^{\frac{3}{2}}}{\left[\left(\Omega_{d}-v\right)^{2}-1\right]^{\frac{1}{2}}} .
$$

Factorizing and re-arranging this equation gives

$$
\hat{\zeta}=\zeta \frac{\left(\frac{\hat{\Omega}_{d}}{\Omega_{d}}\right)\left(1-\frac{\Omega_{u}-\hat{\Omega}_{u}}{\Omega_{u}-1}\right)^{\frac{3}{2}}}{\left(1+\frac{\hat{\Omega}_{d}^{2}-\Omega_{d}{ }^{2}}{\Omega_{d}{ }^{2}-1}\right)^{\frac{1}{2}}}
$$


where $\zeta$ is the true damping ratio given in Eq. (2.7). Eq. (2.11) implies that, unlike the estimate of the cubic stiffness coefficient given by Eq. (2.9), the accuracy of the estimate of the damping ratio depends on the accuracy of both the jumpup frequency and the jump-down frequency.

\section{Experimental investigation}

This section describes the experimental work carried out to determine the jump-up and jump-down frequencies and to further use this information. Thus, the method described in the previous section is verified on a nonlinear electromagnetic energy harvesting device [15]. The device is shown in Fig. 2(a). It is comprised of two main parts. The first part consists of a steel plate of dimensions width $38.21 \mathrm{~mm}$, thickness $0.5 \mathrm{~mm}$, length $42.70 \mathrm{~mm}$ fixed on one edge with a mass of $115 \mathrm{~g}$ and four magnets attached to the opposite edge. The second part of the device is made up of coil wrapped around an iron core. The arrangement of the magnets on the first part is shown in Fig. 2(b).

This arrangement allows the continuous flow of the magnetic flux between the magnets and the iron core. The total stiffness of the system is the combination of the positive stiffness from the plate and the negative stiffness from the magnets resulting in a nonlinear stiffness characteristic. The two main parts are separated by a gap. The gap $d$, which is shown in Fig. 2(a) controls the degree of nonlinearity. When the gap is large the system behaves as a hardening system and when the gap is small the system behaves as a bistable system [16]. In this experiment, the gap was set to $1.5 \mathrm{~mm}$ so that the system behaved as a hardening system.

\subsection{Quasi-static measurement}

The stiffness of the system was estimated by a quasi-static measurement as illustrated in Fig. 3. This was done by attaching the base of the device to an electro-dynamic shaker which was then driven at a very low frequency. The resulting relative displacement between the tip of the plate and the base was measured using a linear variable differential transformer. The force required to keep the tip of the plate stationary was measured using a force gauge, which was attached to ground.

Fig. 4 shows the measured force-deflection plot of the system. The solid curve shows the measured data from the experiment. It can be seen that the plot is not symmetric. This was thought to be due to slight rotation and bending between the connecting arm and the plate. In fitting a polynomial to this curve, symmetry was assumed in which the half cycle where the deflection is positive is mirrored to give a symmetrical curve. This curve was then fitted using the least square method with a cubic polynomial of the Duffing restoring force given by Eq. (1.1), which is shown by the dashed curve in the figure. The stiffness coefficients were found to be $k_{1}=1495 \mathrm{~N} / \mathrm{m}$ and $k_{3}=4.26 \times 10^{7} \mathrm{~N} / \mathrm{m}^{3}$.

\subsection{Dynamic measurements}

For the dynamic measurements, the whole device was placed onto a shaker so that the system was base-excited and the tip of the plate was free. The experimental setup is shown in Fig. 5. The frequency was increased from $15 \mathrm{~Hz}$ to $35 \mathrm{~Hz}$ in $1 \mathrm{~Hz}$ steps and then decreased from $35 \mathrm{~Hz}$ to $15 \mathrm{~Hz}$ with the same frequency increment. Since the system is nonlinear, the amplitude of the input displacement was maintained at a constant value of $0.1 \mathrm{~mm}$ by a feedback controller for all excitation frequencies of interest. The damping in the system was altered by changing the external electrical resistance $R$ connected to the coil using a resistor box. The electrical damping is inversely proportional to the resistance, so that the larger the resistance, the smaller the damping. A PCB accelerometer was used to measure the acceleration of the tip mass, which was recorded together with the input displacement for each frequency.

a

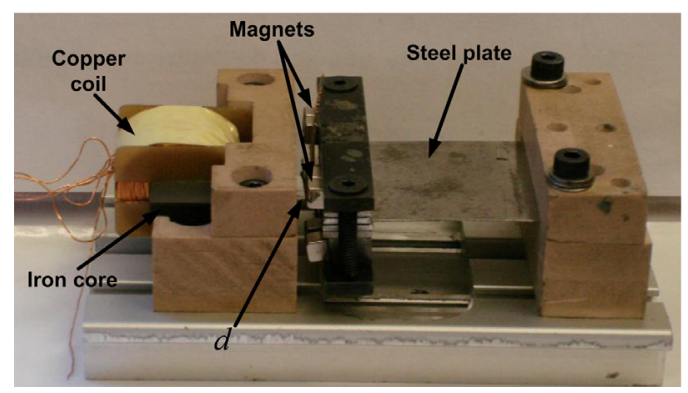

b

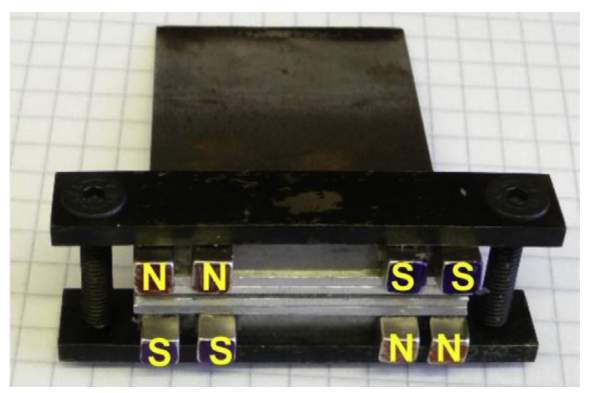

Fig. 2. Photographs showing the experimental device: (a) full view and (b) the arrangement of the magnets. 


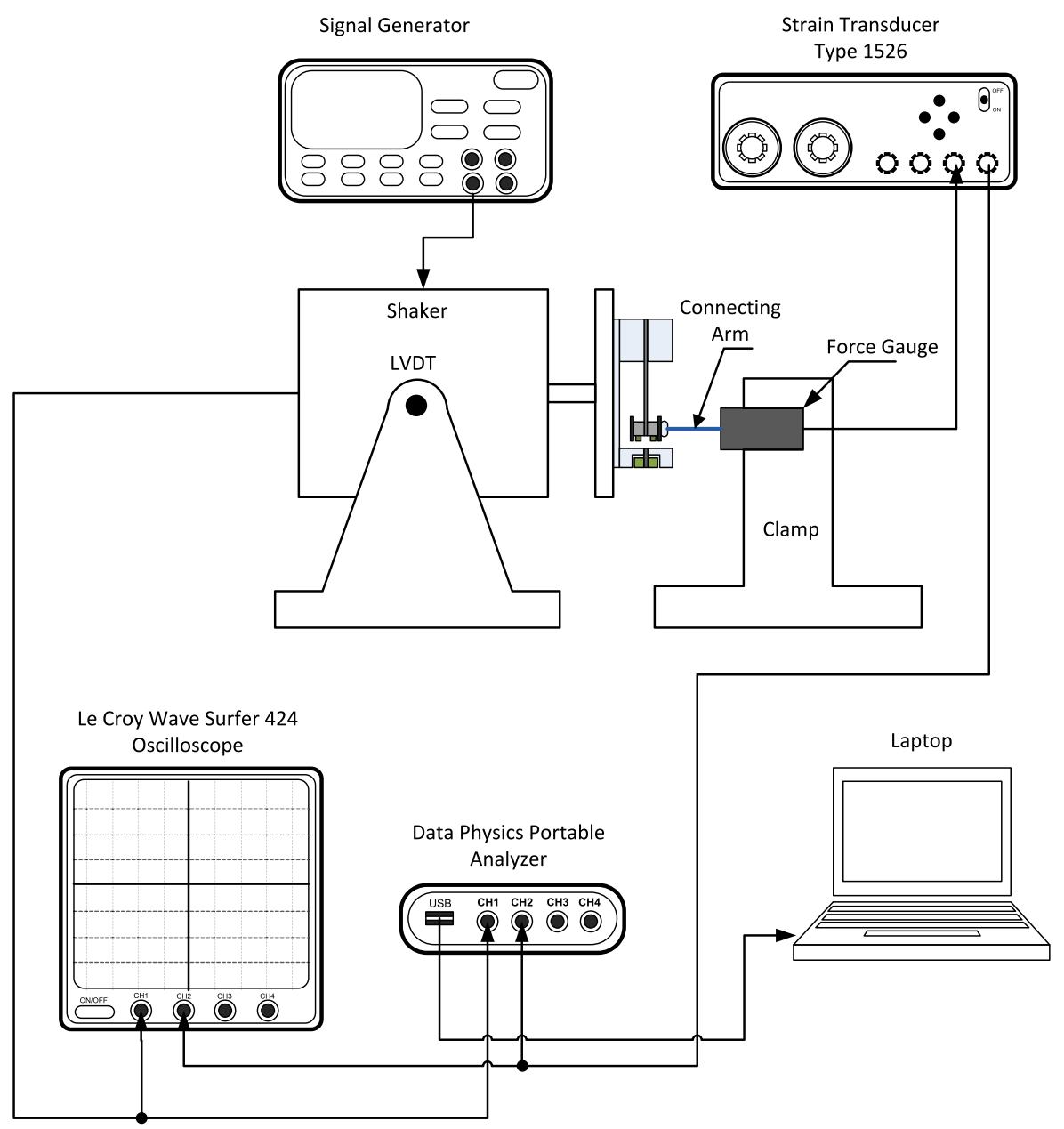

Fig. 3. Experimental setup for quasi-static measurement to determine the stiffness.

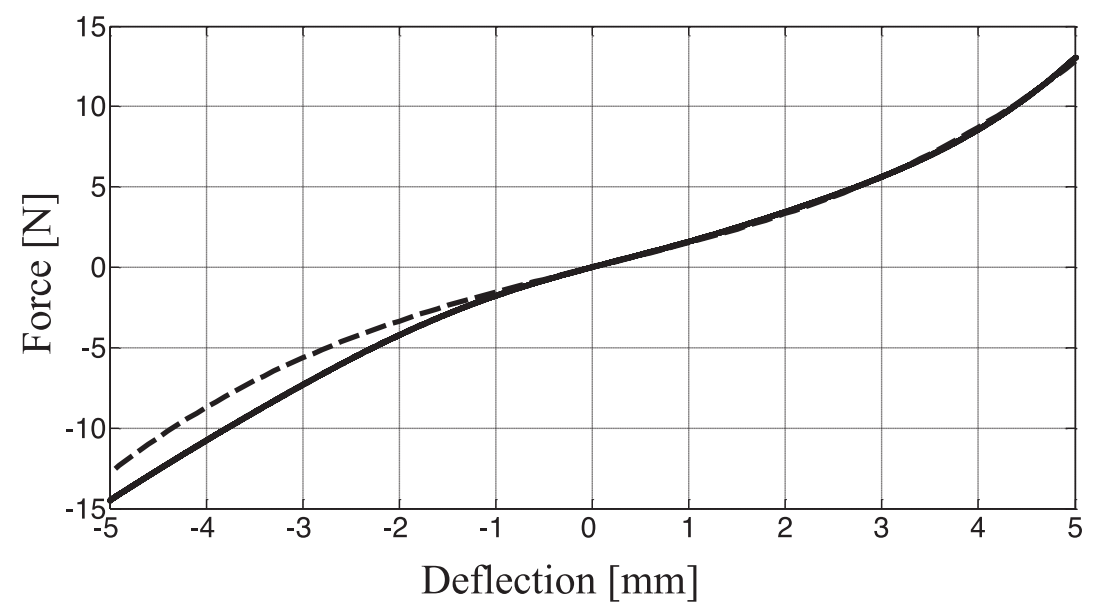

Fig. 4. Force-deflection curve: measured (solid) and fitted by assuming that the system is symmetrical (dashed). 
PCB 442B/04

Sensor Signal Conditioner

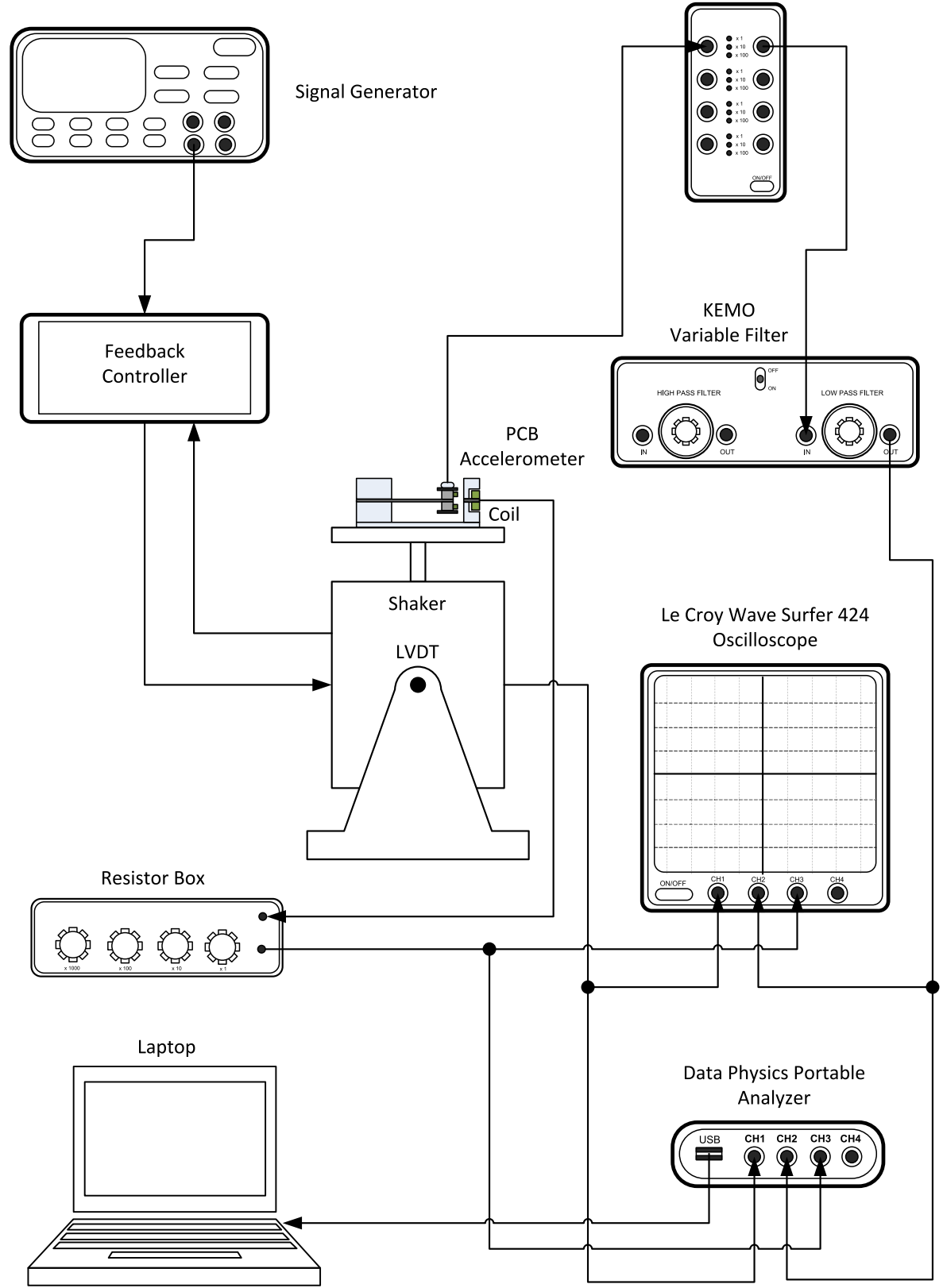

Fig. 5. Experimental setup for dynamic measurements.

\subsection{Comparison between measurements and numerical simulations}

The measured root mean square (rms) displacement for the open-circuit system and that with a $200 \mathrm{Ohm}$ resistance is shown in Fig. 6. It can be seen that the jump-down frequencies lie between $29 \mathrm{~Hz}$ and $30 \mathrm{~Hz}$, and $27 \mathrm{~Hz}$ and $28 \mathrm{~Hz}$ for the open-circuit system (the red solid line with circles) and that with $200 \mathrm{Ohm}$ resistance (the blue solid line with triangles), respectively. However, the jump-up frequency for both configurations (the red dashed line with circles and the blue dashed line with triangles) lies between $26 \mathrm{~Hz}$ and $27 \mathrm{~Hz}$. This supports the observation that the jump-up frequency is only dependent on the cubic stiffness coefficient as given in Eq. (2.4) and the jump-down frequency is a function of both the cubic stiffness coefficient and the damping as given in Eq. (2.5).

To determine the system parameters using Eqs. (2.6) and (2.7), the jump-down frequency for the open-circuit system was taken to be $30 \mathrm{~Hz}$ and for the one with $200 \mathrm{Ohm}$ resistance it was taken to be $28 \mathrm{~Hz}$. The jump-up frequency for 


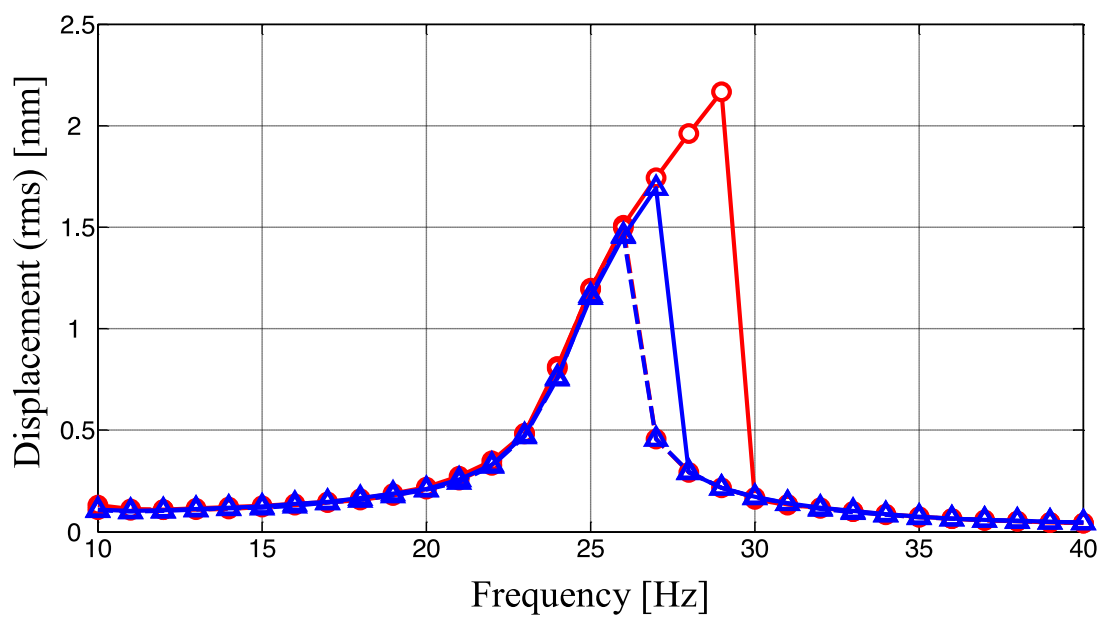

Fig. 6. Measured rms displacement response for the open-circuit system: sweep-up (red-solid-circle), sweep-down (red-dashed-circle). The case of $200 \mathrm{Ohm}$ load is also shown: sweep-up (blue-solid-triangle), sweep-down (blue-dashed-triangle). (For interpretation of the references to colour in this figure legend, the reader is referred to the web version of this article.)

both systems was taken to be $27 \mathrm{~Hz}$. These, of course, are only upper bounds and are chosen based on the $1 \mathrm{~Hz}$ frequency increment. The linear natural frequency was determined experimentally from a measurement of the transmissibility when the device was excited with a low amplitude random input displacement $(0.0054 \mathrm{~mm}(\mathrm{rms}))$ so that the device was still operating in the linear region. These parameters, as well as the cubic stiffness coefficient and the damping ratio calculated using the method described in the previous section, are summarised in Table 1 (note that the frequencies given in Table 1 are dimensional, while for Eqs. (2.6) and (2.7) the non-dimensional values as defined after Eq. (2.2) need to be used).

Fig. 7(a) and (b) show the plots of the simulated and measured responses for the open-circuit system and that with $200 \mathrm{Ohm}$ resistance, respectively. Generally, the simulations and the measurements agree reasonably well. The largest errors occur between $24 \mathrm{~Hz}$ and $26 \mathrm{~Hz}$ for both configurations. This may be due to the error in estimating the jump-up and jump-down frequencies or due to the assumption that only the fundamental harmonic dominates the response with the higher harmonics being neglected (This was checked numerically, and it was found that in some frequency regions, their contributions to the responses are significant; however, these results are not presented here for brevity). It is thought that the presence of the even-order harmonics was because of the asymmetry of the system due to gravity acting on the mass rather than the inherent asymmetry of the stiffness.

The estimate of the cubic stiffness coefficient using the jump-frequencies is also compared to that determined in the quasi-static measurement, which is $\gamma=k_{3} Z^{2} / k_{1}=2.85 \times 10^{-4}$, where $Z=0.1 \mathrm{~mm}$. Comparing this value to the one given in Table 1, it can be seen that the difference between these two estimates is approximately $5 \%$.

The estimate of the damping ratio using the jump frequencies is shown to be accurate as well. It is calculated in three ways: using Eq. (2.7), the logarithmic decrement method [17] and from the peak value of the transmissibility [17]. The three values are plotted in Fig. 8 for several values of the load resistance. Note that the results calculated from the jump frequencies are shown only for the system with electrical resistance greater than $100 \mathrm{Ohm}$ (i.e. small damping). For larger values of damping the system behaves as a linear system with no jump characteristics. Generally, it can be seen that the estimate of damping using the three methods is consistent.

\subsection{Possible errors in the estimation of the non-linearity and the damping parameters}

The sensitivity of the estimated cubic stiffness coefficient and damping ratio on the jump frequencies is studied in this subsection using Eqs. (2.9) and (2.11). Due to the vertical tangency mentioned before, the true values for the jump-up and jump-down frequencies of the system with a $200 \mathrm{Ohm}$ resistance are assumed to be respectively given by $27 \mathrm{~Hz}$ and $28 \mathrm{~Hz}$.

Table 1

Estimated system parameters.

\begin{tabular}{llllll}
\hline Resistance (Ohm) & $\begin{array}{l}\text { Jump-up } \\
\text { freq. (Hz) }\end{array}$ & $\begin{array}{l}\text { Jump-down } \\
\text { freq. (Hz) }\end{array}$ & $\begin{array}{l}\text { Linear natural } \\
\text { freq. (Hz) }\end{array}$ & $\begin{array}{l}\text { Cubic stiffness } \\
\text { coefficient } \gamma\end{array}$ \\
\hline$\infty$ (open-circuit) & 27 & 30 & 25.6 & $2.72 \times 10^{-4}$ & $\begin{array}{l}\text { Damping } \\
\text { ratio } \zeta\end{array}$ \\
200 & 27 & 28 & 25.6 & $2.72 \times 10^{-4}$ & 0.014 \\
\hline
\end{tabular}




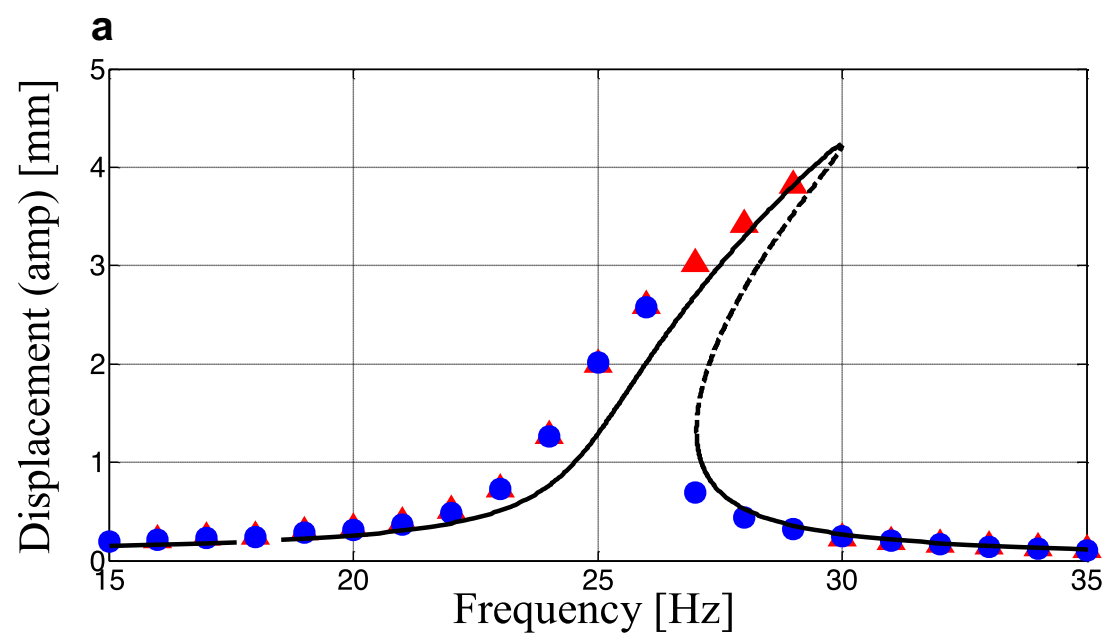

b

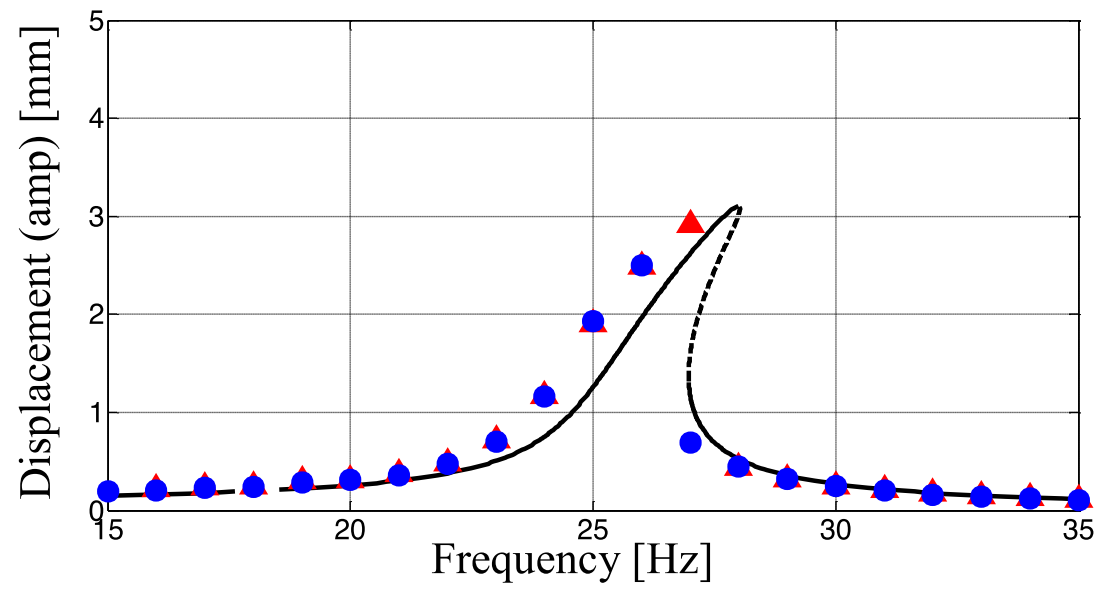

Fig. 7. The simulated response (black solid and dashed lines) and the measured response: red triangle (sweep-up), blue circle (sweep-down); (a) opencircuit and (b) $200 \mathrm{Ohm}$. (For interpretation of the references to colour in this figure legend, the reader is referred to the web version of this article.)

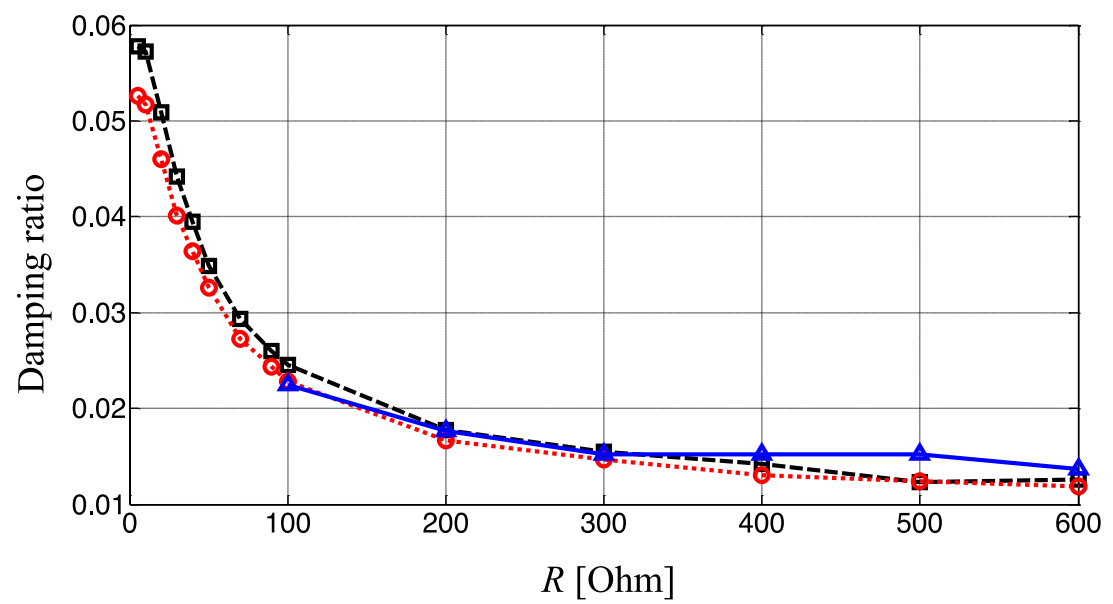

Fig. 8. The estimate of the damping ratio using three different methods; logarithmic decrement method (black-dashed-square), peak of transmissibility (red-dotted-circle), and jump frequencies (blue-solid-triangle). (For interpretation of the references to colour in this figure legend, the reader is referred to the web version of this article.) 


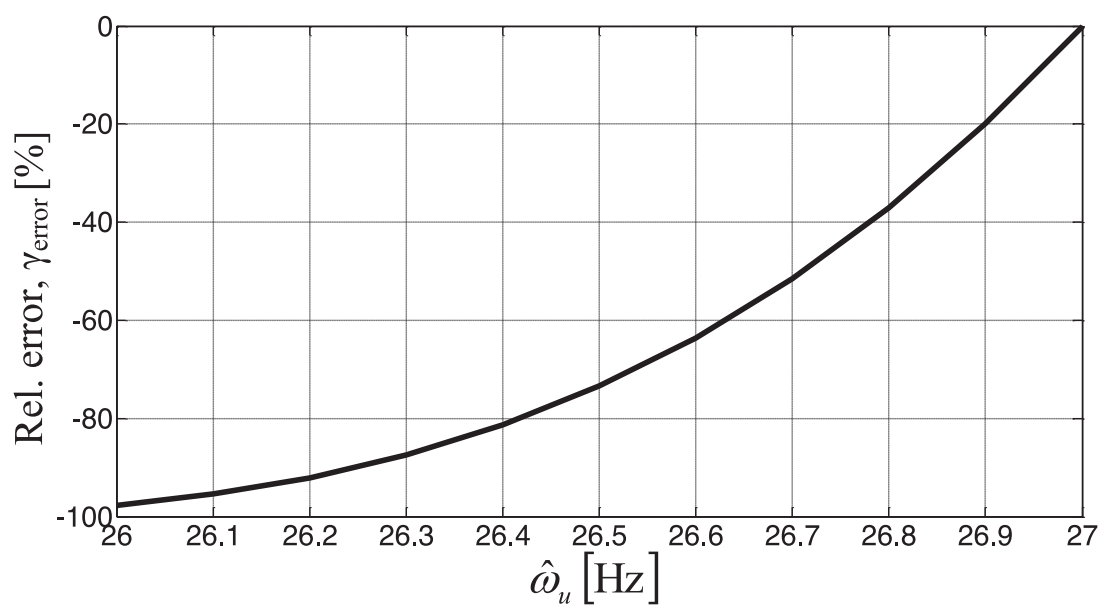

Fig. 9. Relative error in the estimate of non-linearity $\gamma$ with respect to the variation of the jump-up frequency from the assumed true value $27 \mathrm{~Hz}$.

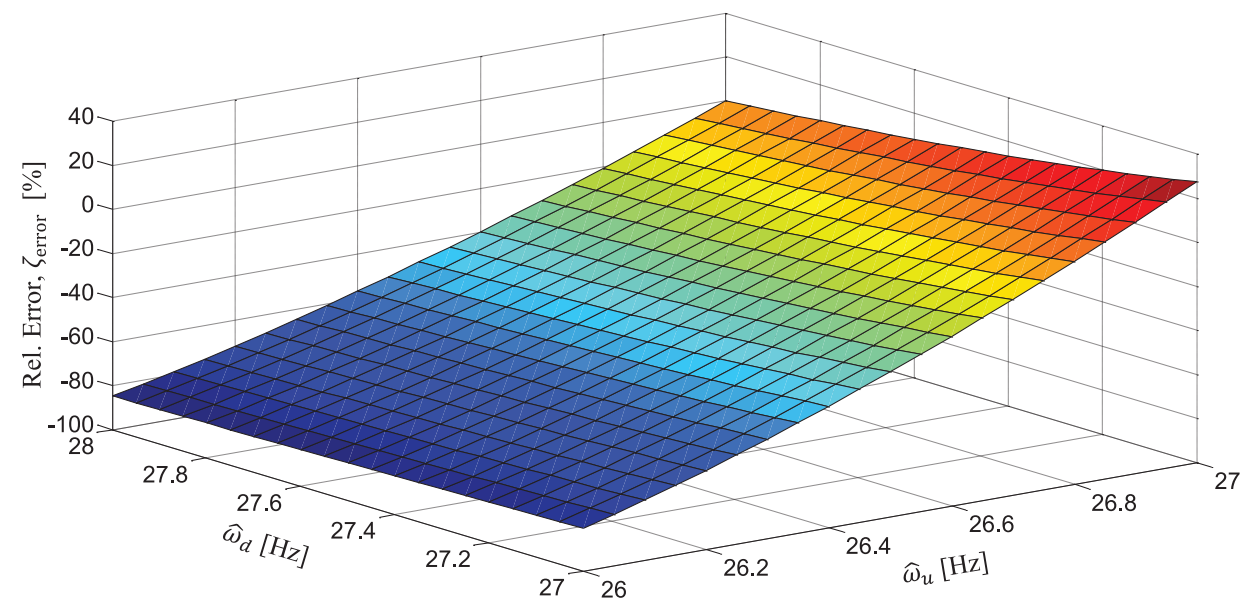

Fig. 10. Relative error in the estimation of the damping ratio for the system with a $200 \mathrm{Ohm}$ resistance with respect to the variations of the jump-up and jump-down frequencies.

The relative error, which may exist when the jump-up frequency deviates away from the assumed true value, is shown in Fig. 9. For this particular configuration, it can be seen that the error in the estimation of the cubic stiffness coefficient can be nonnegligible for a small deviation from the assumed true value of the jump-up frequency.

Fig. 10 shows the relative error in the estimate of the damping ratio with respect to both the jump-up and the jumpdown frequencies for the system with $200 \mathrm{Ohm}$ resistance. It can be seen that the estimate of the damping ratio is less sensitive to changes in the jump-down frequency than the jump-up frequency.

It can be concluded that the error in the estimate of the cubic stiffness coefficient and the damping ratio can be minimised by using a very small frequency increment in the search of the jump frequencies. Thus, for this method to give reliable results, the estimates of the jump frequencies should be as accurate as possible.

\section{Conclusions}

The jump phenomena in a Duffing oscillator are usually seen as undesirable and many techniques and control strategies have been developed to avoid them. However, in this work, it has been shown that knowledge of the jump-up and jumpdown frequencies can be exploited to determine the system parameters. An experiment has been conducted on an electromagnetic device. It has been demonstrated that the estimates of the cubic stiffness coefficient measured quasi-statically and by using the method involving the jump frequencies compared well. The damping in the device has been measured using three different methods including the one involving the jump-frequencies and these also compared well. An error analysis of the proposed method showed that certain errors can potentially occur, but that these can be minimised by using a very small frequency increment in the determination of the jump frequencies. 


\section{Acknowledgement}

Ramlan would like to acknowledge the financial support received from the Ministry of Higher Education Malaysia and Universiti Teknikal Malaysia Melaka through the Bumiputera Academic Training Scheme (SLAB) scholarship award and the Short Term Grant (PJP/2010/FKM (2B) 648).

\section{References}

[1] Wagg D, Neild S. Nonlinear vibration with control. Springer; 2015

[2] Warminski J, Lenci S, Cartmell MP, Rega G, Wiercigroch M, editors. Nonlinear dynamic phenomena in mechanics. Springer; 2012.

[3] Kovacic I, Rand R. Straight-line backbone curve. Commun Nonlinear Sci Numer Simul 2013;18:2281-8.

[4] Rice HJ, McCraith JR. Practical non-linear vibration absorber design. J Sound Vib 1987;116(3):545-59.

[5] Kovacic I, Brennan MJ, Waters TP. A study of a non-linear vibration isolator with quasi-zero stiffness characteristic. J Sound Vib 2008;315(3):700-11.

[6] Liu C, Jing X, Daley S, Li F. Recent advances in micro-vibration isolation. Mech Syst Signal Process 2015;56-57:55-80.

[7] Mann BP, Sims ND. Energy harvesting from the nonlinear oscillations of magnetic levitation. J Sound Vib 2009;319(1-2):515-30

[8] Ramlan R, Brennan MJ, Mace BR, Kovacic I. Potential benefits of a non-linear stiffness in an energy harvesting device. Nonlinear Dyn 2010;59:545-58.

[9] De Paula AS, Inman DJ, Savi MA. Energy harvesting in a nonlinear piezomagnetoelastic beam subjected to random excitation. Mech Syst Signal Process 2015;54-55:405-16.

[10] Younis M. MEMS: Linear and nonlinear statics and dynamics, Springer,

[11] Rega G, Lenci S. A global dynamics perspective for system safety from macro- to nanomechanics: analysis, control, and design engineering. ASME. Appl. Mech. Rev. 2015;67(5):050802-050802-19. doi:10.1115/1.4031705.

[12] Kovacic I, Brennan MJ. The Duffing equation: Nonlinear oscillators and their behaviour. John Wiley \& Sons; 2011.

[13] Brennan MJ, Kovacic I, Carella A, Waters TP. On the jump-up and jump-down frequencies of the Duffing oscillator. J Sound Vib 2008;318(4-5):1250-61.

[14] Tang B, Brennan MJ, Lopes Jr V, da Silva S, Ramlan R, Using nonlinear jumps to estimate cubic stiffness nonlinearity: An experimental study, J Mech Eng Sci. Proceedings of the Institution of Mechanical Engineers Part C, DOI: 10.1177/0954406215606746, in press.

[15] Ramlan R, Brennan MJ, Mace B, Kovacic I, Burrow S. On the estimation of linear viscous damping in the Duffing oscillator. In: Proceedings of the sixteenth international congress on sound and vibration, vol. 2. Kraków, Poland; 2009. p. 1088-95. 5-9 July.

[16] Ramlan R, Brennan MJ, Mace BR, Burrow S. On the performance of a dual-mode non-linear vibration energy harvesting device. J Intell Mater Syst Struct 2012;23:1423-32.

[17] Rao SS. Mechanical vibrations. 2nd ed. Addison-Wesley Pubs; 1990. 